Pereira-García, S.; Devís-Devís, J.; Pérez-Samaniego, V.; Fuentes-Miguel, J.; López-Cañada, E. (2020). The Transsexual and Intersex People in Spanish Competitive Sport: Three Cases. Revista Internacional de Medicina y Ciencias de la Actividad Física y el Deporte vol. 20 (80) pp. 539-551 $\mathrm{Http}: / /$ cdeporte.rediris.es/revista/revista80/artpersonas1186.htm

DOI: https://doi.org/10.15366/rimcafd2020.80.005

\title{
ORIGINAL
}

\section{LAS PERSONAS TRANS E INTERSEXUALES EN EL DEPORTE COMPETITIVO ESPAÑOL: TRES CASOS}

\section{THE TRANSSEXUAL AND INTERSEX PEOPLE IN SPANISH COMPETITIVE SPORT: THREE CASES}

\author{
Pereira-García, S. ${ }^{1}$; Devís-Devís, J. ${ }^{2}$; Pérez-Samaniego, V. ${ }^{3}$; Fuentes-Miguel, \\ J. ${ }^{4}$ y López-Cañada, E. ${ }^{5}$ \\ 1 Profesora Ayudante Doctor, Universidad de Valencia (España) Sofia.Pereira@uv.es \\ ${ }^{2}$ Catedrático Universitario, Universidad de Valencia (España) Jose.Devis@uv.es \\ ${ }^{3}$ Profesor Titular, Universidad de Valencia (España) Victor.M.Perez@uv.es \\ ${ }^{4}$ Profesor Asociado, Universidad de Valencia (España) Jorge.Fuentes@uv.es \\ 5 Dra. en Actividad Física y Deporte por la Universidad de Valencia (España) Elena.Lopez- \\ Canada@uv.es
}

\section{Agradecimientos}

Agradecemos a María José Martínez Patiño la revisión que ha realizado del apartado correspondiente a su caso. María José forma parte del Comité de Expertos de la Comisión Médica del Comité Olímpico Internacional en los temas de hiperandrogenismo y transgénero desde 2012.

Código UNESCO / UNESCO code: 5599 Otras Especialidades: Historia (Historia de la actividad física y el deporte) / Others: History (History of sport and phsyical activity)

Clasificación Consejo de Europa / Council of Europe classification: 07. Historia del deporte / History of sport

Recibido 16 de octubre de 2018 Received October 16, 2018

Aceptado 1 de abril de 2020 Accepted April 1, 2020

\section{RESUMEN}

La concepción cultural del deporte como una actividad predominantemente masculina ha dificultado la participación de algunos grupos sociales como mujeres, personas trans o intersexuales. El carácter sexuado del deporte se apoya en las diferencias fisiológicas entre mujeres y hombres, y una supuesta desventaja de las mujeres. Por ello, se establecen pruebas de sexo para las mujeres y el acceso de las personas trans e intersexuales se ve obstaculizado. 
En este estudio reconstruimos, a partir de las normativas y el contexto sociohistórico internacional, la evolución de la participación de personas trans e intersexuales en el deporte competitivo contemporáneo. Asimismo, se profundiza en la aplicación y gestión de dichas normas en el contexto español, apoyado en tres casos de deportistas trans e intersexuales españoles. La discriminación y humillación que han sufrido estas personas obliga a mantener una visión crítica de las políticas deportivas creadas hasta la actualidad.

PALABRAS CLAVE: trans, intersexual, deporte, normativas, inclusión

\section{ABSTRACT}

The cultural conception of sport as a predominantly male activity has hindered the participation of some social groups such as women, transsexual or intersex people. The sexed nature of sport is based on physiological differences between women and men, and a supposed women disadvantage. Thus, sex controls are established for women and then trans and intersex people's access to sport is hampered. In this study we reconstruct, based on the regulations and the international socio-historical context, the evolution of the participation of transsexual and intersex people in contemporary competitive sport. Likewise, the application and management of these norms in the Spanish context is deepened, supported by three cases of Spanish transsexual and intersex athletes. The discrimination and humiliation suffered by these persons enforce to maintain a critical vision of the sports policies hitherto created.

KEY WORDS: transsexual, intersex, sport, regulations, inclusion

\section{INTRODUCCIÓN}

Las dificultades y obstáculos que han experimentado las personas trans e intersexuales que deciden participar en la práctica deportiva, especialmente a un alto nivel, han estado conectadas con los debates sobre el dopaje deportivo y las pruebas de sexo que provienen desde los tiempos de la Guerra Fría. Como veremos en las páginas de este trabajo, la manera en que se han interrelacionado estos dos temas ha ido dando forma al entorno cultural y normativo internacional actual sobre la participación de las personas trans e intersexuales en el deporte (Reeser, 2005; Ritchie, 2003).

Durante los últimos años, la delimitación de qué es o qué no es dopaje ha generado una considerable controversia. Esto es así porque se han permitido algunas sustancias farmacológicas o nutricionales que pueden mejorar el rendimiento en determinados momentos y poco después se han prohibido, llamando 'tramposos' a los deportistas que las han consumido posteriormente. Pero la controversia se ha extendido a otras situaciones que afectan a algunas mujeres y a personas trans e intersexuales. 
Sabemos que la administración de hormonas androgénicas, como la testosterona, produce una ventaja deportiva significativa debido al aumento de la fuerza y la resistencia de las personas que las toman (Karkazis, Jordan-Young, Davis y Camporesi, 2012). En el caso de personas trans o intersexuales, la administración externa de estas sustancias puede ser necesaria para alcanzar o mantener sus identidades de género. Sin embargo, los motivos que se encuentran detrás de la decisión de consumirlas no se tienen en cuenta por los organismos internacionales que sancionan, igualmente, estas conductas. Además, no sólo se penaliza a quienes consumen estas hormonas sino también a deportistas que generan altos niveles de testosterona de manera natural, tal y como ha ocurrido recientemente con Caster Semenya (Amorós, 2019; Buzuvis, 2010).

La vigilancia sobre las mujeres deportistas no es algo nuevo. Desde que comenzaron a participar en el deporte, un campo que originalmente ha marcado como legítimo, meritorio y deseable lo masculino (Monforte y Úbeda-Colomer, 2019), sus cuerpos se han sometido constantemente al examen y escrutinio médico-científico. Así lo demuestra el largo historial de pruebas desarrolladas para descubrir, mediante test 'científicos', si eran hombres farsantes que pretendían hacerse pasar por mujeres para obtener ventaja deportiva en las competiciones. Estas pruebas han tenido el poder, no solo para determinar si las mujeres podían competir o no, sino también para comprobar si las mujeres eran mujeres 'verdaderas' o no. Aquellas que no superaban estas pruebas eran expulsadas de la competición y consideradas anormales. Sin embargo, las consecuencias éticas de las pruebas de sexo todavía están sin asumirse. Por ello, se ha generado un debate social y político que cuestiona el mito de la justicia competitiva que mantiene la necesidad de las pruebas de sexo (Buzuvis, 2010; Karkazis, Jordan-Young, Davis y Camporesi, 2012). Este debate también se extiende a la evaluación científica del sexo únicamente entre dos categorías mutuamente excluyentes de hombres y mujeres (Sullivan, 2011).

A pesar de la creciente preocupación internacional sobre el dopaje, las pruebas de sexo y la participación deportiva de las personas trans e intersexuales, apenas se conocen las circunstancias que han rodeado la participación deportiva de estas personas en el último siglo en España. Por lo tanto, el objetivo principal de este artículo es reconstruir cómo ha evolucionado la participación de personas trans e intersexuales en el deporte competitivo, a través del análisis documental de las circunstancias personales y socioculturales que acompañan los casos de Torremadé, Patiño y Parés, tres deportistas de la historia reciente en España.

\section{El caso Torremadé y la obsesión por los hombres tramposos}

María Torremadé, nacida en Barcelona en 1923, jugó a baloncesto y hockey, obteniendo resultados brillantes con tan solo 18 años. En febrero de 1942, un periódico español informó que iba a someterse a un 'cambio de sexo' [sic.] porque se sentía un hombre, convirtiéndose en Jordi desde entonces. En el momento en que se hizo pública esta noticia, la extraordinaria carrera deportiva de 'aquella mujer' se vino abajo (García Candau, 2009). Algunos años antes, el ciclista belga Willy de Bruyne, el campeón británico de lanzamiento de peso y jabalina Mark Weston y el corredor checoslovaco Zdenk Koubkov, que 
desarrollaron sus carreras deportivas como mujeres, vivieron situaciones similares (Bilharz, 2005; Meyerowitz, 1998). En todos estos casos, las sospechas de ser 'marimachos' durante su carrera deportiva provocaron reacciones sociales negativas a estos deportistas desde los medios de comunicación y la sociedad en su conjunto.

Parecer o ser 'marimacho' fue, y sigue siendo, un problema real para las mujeres que compiten en el deporte. Desde que las mujeres comenzaron a participar en el deporte de élite, ha habido una preocupación acerca del engaño de hombres deportistas que competían en categorías femeninas (Hargreaves, 1994). Esta preocupación se basó en la suposición de que era más fácil para los hombres ganar dentro de las competiciones femeninas, debido al menor rendimiento 'natural' de las mujeres. Por ello, las instituciones deportivas empezaron a regular la participación femenina a través de pruebas de sexo durante las décadas de 1930 y 1940 . Estas pruebas recibieron un fuerte impulso por el caso de 'Dora' Ratjen, un hombre alemán que participó como mujer en la prueba de salto de altura femenino en los Juegos Olímpicos de Berlín, en 1936. Ratjen, que compitió obligado por el nazismo para excluir de la competición a la atleta alemana judía Gretel Bergmann, fue acusado de tramposo y 'hermafrodita'. Pero su caso sirvió para justificar las estrictas medidas posteriormente adoptadas por las instituciones deportivas (Buzuvis, 2010; Heggie, 2010; Karkazis, JordanYoung, David y Camporesi, 2012; Leal, 2014; Sullivan, 2011).

Sin embargo, el síndrome de Torremadé, un tipo de intersexualidad caracterizada por tener una apariencia de genitales externos femeninos, pero con una carga genética de cromosomas XY (síndrome de Morris), no se encontró a través de una prueba sexual (Ladrón de Guevara, 2015). Por lo tanto, el 'cambio de sexo' de Torremadé fue una decisión exclusivamente personal. No obstante, los medios de comunicación consideraron que se trataba de una práctica engañosa que confirmaba las sospechas sobre su masculinidad y, en consecuencia, el fraude durante sus años de competición. Las instituciones deportivas españolas anularon sus registros deportivos de forma inmediata.

El caso de Torremadé debe entenderse dentro de los contextos socioculturales, tanto nacionales como internacionales en los que se produjo. En la España franquista, el deporte estaba controlado por uno de los principales aparatos ideológicos de Estado, el Frente de Juventudes. Para la rama femenina de esta organización, la Sección Femenina, el verdadero papel de las mujeres era el de convertirse en madres y la práctica deportiva se consideraba que favorecía la masculinización de sus cuerpos (Machado y Fernández, 2015). Únicamente se contemplaba adecuada la actividad física y el deporte para las niñas y jóvenes con el único objetivo de fortalecer su cuerpo y así cumplir con sus posteriores deberes en el matrimonio (Manrique, 2018). Por lo tanto, "ser un referente deportivo no era un valor añadido a la mujer" (Manrique, 2014: 440) sino todo lo contrario. Además, para Pilar Primo de Rivera, representante nacional de la Sección Femenina, el caso de Jordi Torremadé sirvió para reforzar y apoyar la idea de mantener a las mujeres alejadas del deporte (Ródenas, 2014). De hecho, la Sección Femenina pronto prohibió la competición en actividades deportivas a todas las mujeres hasta principios de la década de 1960 (García Candau, 2009). 
Este caso acontece durante el auge internacional por la detección de hombres 'tramposos' que mostraron las organizaciones deportivas durante las décadas de 1940 y 1950. De hecho, la Asociación Internacional de Federaciones de Atletismo (IAAF) implantó la política de pruebas de sexo en 1946 y el Comité Olímpico Internacional (COI) hizo lo mismo dos años después. El nuevo reglamento requería un examen obligatorio a todas las deportistas por parte del personal médico que verificaba su sexo biológico y detectaba a posibles hombres encubiertos (Heggie, 2010; Karkazis, Jordan-Young, Davis y Camporesi, 2012). El examen requería que cada mujer estuviera desnuda frente a los expertos médicos que comprobaban sus genitales. Esta medida desalentó a algunas deportistas que decidieron retirarse o simularon lesiones para evitar el vergonzoso y humillante proceso de verificación. Más allá de la detección de deportistas deshonestos, la prueba estaba destinada a determinar y definir qué era una mujer 'verdadera'. Curiosamente, las organizaciones deportivas internacionales estaban mucho más preocupadas por el engaño de las 'mujeres falsas' que por las prácticas de dopaje. Según Hoberman (2005), durante la década de 1950, las organizaciones deportivas y el público en general se mostraron más permisivos con deportistas, especialmente hombres, que usaban drogas para mejorar su rendimiento, mientras que cada vez más mujeres eran vigiladas por estos controles de sexo.

\section{El caso Patiño y la sofisticación de las pruebas de sexo}

Durante la segunda mitad del siglo XX, las pruebas de sexo adoptaron técnicas médicas más sofisticadas. En 1967 la IAAF instauró unas pruebas cromosómicas de sexo (análisis genético de los 'cuerpos de Barr') que reemplazaban la evaluación anatómica y se convirtieron en obligatorias hasta finales de 1990. Los avances científicos dieron lugar a nuevas pruebas como la técnica PCR (polymerase chain reaction) que sustituyeron a las pruebas anteriores. En la década de 1980, la comunidad médica comenzó a cuestionar el uso de estas pruebas, y una década más tarde, la IAAF reconoció ciertas limitaciones y se detectaron varios fraudes. Lo cierto es que desde que comenzaron las pruebas de sexo no se encontró a ningún hombre tramposo (Sullivan, 2011). Por el contrario, estas pruebas tuvieron éxito en la detección de mujeres intersexuales o no normativas (conocidas por los médicos como mujeres con 'trastornos del desarrollo sexual adecuado').

María José Martínez Patiño fue la primera atleta que falló en la prueba cromosómica durante los exámenes realizados en la Universiada de Kobe, en 1985. Patiño, que destacó en las pruebas de 60 y 100 metros vallas, estaba preparándose para participar en los siguientes Juegos Olímpicos de Seúl cuando los médicos le informaron de su alteración cromosómica (Buzuvis, 2013). El equipo oficial de Kobe le aconsejó que simulara una lesión, pero ella rechazó la propuesta. La Real Federación Española de Atletismo la envió de regreso a España donde fue sometida a nuevas pruebas. Los resultados de estas pruebas confirmaron un diagnóstico de insensibilidad completa a los andrógenos y una ratificación de su condición genética $X Y$. Entonces le sugirieron que se retirara de la competición con discreción. Ante su negativa y la victoria en una competición nacional en 60 metros vallas, su informe médico se filtró a los medios de comunicación. La protección a la intimidad, derivada de la 
confidencialidad médica, no se respetó en su caso. Además, la Real Federación Española de Atletismo le expulsó de la residencia Blume de Madrid en la que residía, eliminó todo su apoyo financiero y todas las marcas obtenidas hasta ese momento (Hernández, 2014; Parks, 2014).

En esa época, España había estrenado un sistema democrático después de un período de transición. Se observaba un repunte importante en la participación deportiva de la ciudadanía (Machado y Fernández, 2015; Ródenas, 2014), aunque la participación de las mujeres era sustancialmente menor que la de los hombres. Ya no existía un equivalente a la Sección Femenina del franquismo y tampoco un contexto ideológico en contra de la participación deportiva de las mujeres. La participación de las mujeres incrementó en todas las esferas del deporte y, en especial, en el deporte de élite (Leruite, Martos y Zabala, 2015), donde las competiciones deportivas eran vistas por buena parte de la sociedad como un signo de modernidad y una forma más de participación de la mujer en la democracia española. Sin embargo, a nivel internacional, el aumento espectacular del uso de drogas artificiales para mejorar el rendimiento deportivo alimentó la preocupación sobre las pruebas de sexo, afectando al control de la vida privada de las mujeres y su participación deportiva. Así se acrecentó la presión de las instituciones deportivas sobre las mujeres que generaban altos niveles de testosterona de manera natural. Esto era debido a que estas mujeres se homologaban con deportistas dopados y también se les acusaba, socialmente, de sacrificar su feminidad para obtener la victoria en la competición (Dimeo, 2007; Gleaves, 2015).

En este contexto, Patiño demandó a la Real Federación Española de Atletismo y luchó contra la opinión pública para volver a las competiciones de atletismo. Buscó fuera de España el apoyo que en su país se le había negado hasta que la Comisión Médica de la IAAF, con el apoyo de su presidente, el profesor Arné Lungqvist y del genetista finlandés Albert de La Chapelle, reabrió su caso. En una reunión de la Comisión, celebrada en Seúl con motivo de los Juegos Olímpicos, se determinó que su condición biológica no le daba ninguna ventaja ilegal y que podía competir de nuevo.

Desafortunadamente, después de tres años, rodeada del barullo mediático y miradas llenas de sospecha, el rendimiento deportivo de María José se había resentido enormemente y las consecuencias negativas para su carrera deportiva y vida personal ya fueron irreversibles. Como muchas otras mujeres deportistas, no solo fue víctima de una regulación restrictiva y de una opinión pública inquisidora, sino también del rígido sistema binario sexo-género que deslegitimaba y marginaba aquellos cuerpos que excedían los límites definidos por la normalización.

Desde entonces, los organismos deportivos nacionales e internacionales han flexibilizado sus políticas deportivas sobre las pruebas de sexo y la participación de deportistas intersexuales. El caso de Patiño contribuyó a cambiar las pruebas de sexo basadas en un análisis cromosómico, utilizadas solo con las mujeres deportistas y no con los hombres. Acabó por replantear las normativas y la condición de las mujeres intersexuales en el ámbito del deporte de competición. Con el tiempo se evolucionó hacia el control de rangos de normalización de 
testosterona en sangre, tanto para mujeres como para hombres, aunque en este último caso por razones de dopaje (García Dauder, 2011). Pero este cambio no afectó al predominio del discurso médico en las decisiones y criterios aplicados por las organizaciones deportivas. Ya en 2003, el COI acordó estudiar caso por caso los altos niveles de testosterona en las mujeres y la IAAF adoptó este mismo criterio tres años más tarde (Hercher, 2010). De esta manera, solo las atletas 'sospechosas' eran examinadas con una evaluación médica multidimensional y profunda. No obstante, como indican Cooky y Dworkin (2013: 108), en la práctica "las mujeres se someten a pruebas de sexo cuando tienen un rendimiento atlético explosivo, un alto grado de musculatura o se perciben como 'demasiado masculinas'”.

Caster Semenya ha sido, probablemente, el caso de intersexualidad en el deporte que mayor repercusión internacional y mediática ha tenido. Cuando ganó la prueba de 800 metros en el Campeonato Mundial de Atletismo de 2009 se le cuestionó su feminidad debido al destacado rendimiento deportivo que obtuvo. Su desarrollada musculatura y grave voz también contribuyeron a que se extendiera rápidamente esa desconfianza. De hecho, una comisión médica le solicitó que se sometiera a un procedimiento de verificación de sexo ese mismo año (Buzuvis, 2010). La IAAF esperó a julio de 2010 para comunicar la conclusión de dicha comisión en la que se decía que Semenya podía competir como mujer, a pesar de que su cuerpo segregaba, de manera natural, más testosterona que los rangos 'normales' establecidos para las mujeres. La normativa aplicada durante los Juegos Olímpicos de Londres de 2012 indicaba que el rango de testosterona debería de estar por debajo de 10 nanomoles por litro de sangre. Así que siguió compitiendo y ganando carreras.

Sin embargo, en abril de 2018, la IAAF cambió el criterio sobre la elegibilidad para la competición de las atletas con altos niveles de testosterona. El nuevo reglamento indicaba que, si la concentración de testosterona superaba los 5 nanomoles por litro, la atleta debe seguir un tratamiento hormonal para rebajarla (IAAF, 2018). Esta norma provocaba lo que se ha denominado 'dopaje inverso' por D'Angelo y Tamburrini (2013), que resultó ser muy criticada por ser irrespetuosa con las deportistas y potencialmente peligrosa. A pesar de ello, a las mujeres con niveles superiores a los 5 nanomoles se les prohibió competir en carreras de medio fondo (las correspondientes a distancias que van de 400 metros a la milla), precisamente en las que destacaba Semenya.

Actualmente, el caso de Semenya todavía se encuentra en los tribunales y ella continúa rechazando rotundamente someterse a medicación por unas reglas que considera discriminatorias. Hasta que se resuelva, la atleta ha fichado por el JVW Fútbol Club, un equipo de la liga de fútbol femenino de Sudáfrica con el que jugará mientras tanto, aunque sin dejar de lado completamente el entrenamiento del atletismo (Amorós, 2019).

Otro caso que se hizo popular en 2014 fue el de Dutee Chand, especialista en las pruebas de velocidad de 100 y 200 metros. A esta atleta de 18 años se le suspendió la práctica del atletismo por detectarle hiperandrogenismo o exceso de andrógenos. Su batalla jurídica le llevó hasta el Tribunal Arbitral del Deporte (TAS) en 2015 y ganó el derecho a competir de nuevo. Chand también rechazó 
tomar píldoras o realizar operaciones quirúrgicas para alcanzar los valores de andrógenos que le permitieran competir. Actualmente, como la nueva regulación afecta a distancias más largas, Chand no tiene que someterse a ninguna medicación y puede seguir compitiendo con normalidad. Sin embargo, no es un caso que cause grandes controversias desde el punto de vista normativo porque sus resultados deportivos son modestos.

\section{El caso Parés y las personas trans}

Los impedimentos para competir no sólo han afectado a las personas intersexuales. Las personas trans también han sido tradicionalmente rechazadas en el deporte, especialmente las mujeres trans porque se perciben como deportistas con una ventaja competitiva injusta. Aunque cada deportista trans es tratada con sospecha, las organizaciones deportivas no aplican el criterio caso por caso utilizado con participantes intersexuales. Como consecuencia, la mayoría de estas personas abandonan sus carreras deportivas o retrasan su proceso de transición para competir durante algunos años más, antes de hacer pública sus identidades de género.

El primer caso conocido de transexualidad en el deporte español es el de Natalia Parés, jugadora de ajedrez. Desde mediados de la década de 1970 hasta finales de la de 1990 obtuvo grandes éxitos nacionales con el nombre de Josep. Además, recibió el título de Maestro de Ajedrez por la Federación Internacional, pero se retiró cuando reveló públicamente su identidad de género en 1998. Como no podía modificar su nombre en el registro civil porque aún no era posible por ley (se aprobó la Ley 3/2007 el 15 de marzo), no podía participar como mujer. Desde entonces, estuvo involucrada en el activismo trans para sensibilizar a la sociedad sobre los derechos de las personas trans y las dificultades para su inclusión (Boyero, 2006; La Vanguardia, 2008).

Seis años después de la retirada de Natalia, el COI se convirtió en la primera institución que regulaba el acceso de las personas trans en la competición. En ese momento, se establecieron tres condiciones para que las personas trans (especialmente mujeres trans) pudieran competir: 1) cirugía de reasignación de sexo; 2) el reconocimiento legal de la nueva identidad de género; y 3) al menos dos años de tratamiento hormonal posterior a la cirugía si se había producido después de la pubertad (COI, 2003).

En los últimos años, el crecimiento y fortalecimiento del activismo trans y la mayor visibilidad de estas personas en diferentes ámbitos sociales también se ha filtrado en el contexto deportivo. Además, la defensa de los derechos de las deportistas afectadas por las pruebas de sexo en deportes competitivos (por ejemplo, Heidi Krueger o Tamara e Irina Press), han provocado cambios en las regulaciones deportivas, reconociendo cada vez más su derecho a participar en las competiciones deportivas. Así, desde el año 2015, las personas trans pueden participar en el deporte de élite sin someterse a una cirugía de reasignación de sexo, aunque todavía se requiere el reconocimiento legal y el tratamiento hormonal para competir (COI, 2015). 
El COI llegó a esta situación después de que varias deportistas trans reivindicaran, con muchas dificultades y rechazos por parte de la sociedad, su derecho a participar. El caso internacional más famoso fue el de Renée Richards, una jugadora trans de tenis que en 1977 logró participar en el US Open en la categoría femenina tras realizarse una vaginoplastia y ser reconocida legalmente como mujer. Otras deportistas y activistas trans, como las golfistas Mianne Bagger y Lana Lawless, así como las ciclistas Michelle Dumaresq y Kristen Worley, también realizaron grandes esfuerzos para ser incluidas en sus respectivos deportes (Cascardo, 2019).

Ante la situación, Natalia Parés fue animada por su entorno cercano para volver a la competición. Con las tres condiciones del COI, Natalia obtuvo el título de Maestra de Ajedrez, ya como mujer, ganó la segunda posición en el Campeonato de España de Ajedrez Femenino de 2008 y formó parte del equipo femenino español. A pesar de su exitosa vuelta, Natalia vio afectado negativamente su rendimiento por esos 10 años de retirada forzada en los que dejó de lado su carrera deportiva.

Recientemente se han hecho públicos nuevos casos de deportistas trans que participan en competiciones organizadas en España, lo que refleja una mayor visibilidad e inclusión de estas personas en entornos deportivos. Uno de ellos es Oscar Sierra, un joven jugador de fútbol americano a quien se le permitió jugar en un equipo masculino en la tercera división durante la temporada 2015-2016. La Federación Española de Fútbol Americano y el Consejo Superior de Deportes aprobaron su solicitud, a pesar de que no cumplía con los dos años de tratamiento hormonal establecido en la normativa del COI.

Por otro lado, Antia se convirtió en la primera mujer transexual en debutar en una competición oficial española en un deporte olímpico, el voleibol. La Federación Española de Voleibol y su club, Calasancias, le dieron la tan esperada ficha deportiva federada que reconocía su derecho a competir en 2015. Otro caso actual conocido es el de Izaro Antxia, que en 2016 se convirtió en la primera jugadora transexual de fútbol sala federada en España. A pesar de su buena acogida, Izaro sigue soportando acoso e insultos en algunos de los encuentros que disputa. Desde el último trimestre de 2018, las personas trans también pueden participar en competiciones ciclistas. Gracias a esta normativa, Gael, un chico trans ciclista, ya puede competir en la categoría de élite masculina como federado. Llevaba siete años haciéndolo en la categoría de élite femenina, pero la Real Federación Española de Ciclismo (RFEC) ha establecido un protocolo que reconoce y facilita la competición de las personas trans, desde el respeto a su identidad y favoreciendo la igualdad para todas las personas (Rivera, 2019).

A pesar de las nuevas políticas que amplían los derechos de participación de deportistas trans e intersexuales, la regulación sigue siendo rígida. La comunidad científica, las y los deportistas trans e intersexuales, y la sociedad en general, todavía tienen importantes retos para eliminar algunas prácticas y políticas discriminatorias y poco éticas que afectan a estas personas. 


\section{CONCLUSIONES}

Más allá del interés personal, las historias de deportistas españoles trans e intersexuales como Jordi Torremadé, Mํㅡ José Martínez Patiño y Natalia Parés, reflejan la evolución sociopolítica del entorno nacional e internacional del deporte. Las organizaciones deportivas internacionales más relevantes como el COI o la IAAF han ejercido el poder de considerar qué es justo o injusto en la competición deportiva a lo largo del tiempo. Incluso se han otorgado el derecho de decidir quién es o no una mujer, al menos en el contexto deportivo.

Contrariamente a lo que sucedía a principios del siglo XX, actualmente las políticas deportivas son más flexibles, y un mayor número de deportistas trans e intersexuales participan en competiciones deportivas. Incluso han evolucionado y se han sofisticado las pruebas de verificación de sexo. Sin embargo, todavía hoy en día, tal y como ocurría a principios del siglo XX, las mujeres siguen siendo las únicas personas que se someten a este tipo de pruebas. Como indica Hercher (2010), las autoridades deportivas deben abstenerse de determinar quién es y quién no es una mujer o un hombre, ya que se trata más de una cuestión ética que médica. Buzuvis (2013), por su parte, señala que "lo que dificulta esta clasificación es que, si bien el mundo del deporte divide el universo en categorías masculinas y femeninas, la naturaleza no" (p.55). En este sentido, los cuerpos de personas trans e intersexuales subvierten los límites biológicos del sexo, lo que problematiza el mito del sexo binario y la organización deportiva basada en un sexo 'claro'.

La regulación de la justicia deportiva es intrínsecamente compleja. No hay ninguna razón científica por la cual el hiperandrogenismo deba tratarse de manera diferente a otras diferencias genéticas que confieren un mayor rendimiento deportivo. Más allá del sexo, otras variables como ser más alto o tener más equilibrio que el promedio no se tienen en cuenta por las organizaciones deportivas y podrían considerarse igualmente relevantes para garantizar una competición justa (Buzuvis, 2013; Hargreaves, 1994). Un ejemplo claro es el del nadador Michael Phelps cuya anatomía se caracteriza por tener pies anormalmente grandes y tobillos hipermóviles que actúan como aletas. Además, sus extremidades superiores son más largas de lo normal mientras que las inferiores son relativamente más cortas, reduciendo así la resistencia en el agua (Cooper, 2010). En este sentido, ¿por qué el sexo debe ser el pilar que mantiene la construcción de la supuesta injusticia en las competiciones deportivas?

Las regulaciones sobre la participación de personas trans e intersexuales en el deporte deberían considerar no sólo las cuestiones relacionadas con la justicia competitiva, como las indicadas anteriormente, sino también el sufrimiento de aquellas personas cuyas identidades de género han sido constantemente escudriñadas, cuestionadas e ignoradas. Es necesario, por tanto, tener en cuenta las consecuencias éticas de las normativas para proteger la integridad moral de las y los deportistas trans e intersexuales. 


\section{REFERENCIAS BIBLIOGRÁFICAS}

Amorós, A. (9 de septiembre, 2019). El fútbol, un refugio para Semenya. $A B C$ Deportes. Recuperado de: https://www.abc.es/deportes/abci-futbolrefugio-para-semenya-201909090353 noticia.html

Bilharz, J. (2005). Paradigms lost: Cultural and legal implications of sexual ambiguity. Forum of Public Policy 1(3), 247-258. Recuperado de: http://forumonpublicpolicy.com/vol1.no3.gender/bilharz.pdf

Boyero, J. (4 de marzo, 2006). El ajedrez español no sabe de sexos. Marca. Recuperado de: http://escacs.cat/premsa/marcamarzo4.pdf

Buzuvis, E. (2010). Caster Semenya and the myth of a level playing field. The Modern American, 6(2), 36-42.

Buzuvis, E. (2013). Transsexual and Intersex Athletes. In M. Sartore-Baldwin (ed.), Sexual Minorities in Sports: Prejudice at Play (pp. 55-71). Boulder: Lynne Rienner Publishers.

Cascardo, M. (2019). Deportistas transgénero por el mundo. ElEquipo. Recuperado de: https://elequipo-deportea.com/2019/03/07/deportistastransgenero-por-el-mundo/

COI (Comité Olímpico Internacional). (2003). Statement on the Stockholm Consensus on sex reassignment in sport. Recuperado de: https://stillmed.olympic.org/Documents/Reports/EN/en_report_905.pdf

COI (Comité Olímpico Internacional). (2015). IOC Consensus meeting on sex reassignment and hyperandrogenism. Retrieved from: https://stillmed.olympic.org/Documents/Commissions_PDFfiles/Medical_co mmission/2015-

11_ioc_consensus_meeting_on_sex_reassignment_and_hyperandrogenis m-en.pdf

Cooky, C. y Dworkin, S. (2013). Policing the Boundaries of Sex: A Critical Examination of Gender Verification and the Caster Semenya Controversy. Journal of Sex Research, 50(2), 103-111. https://doi.org/10.1080/00224499.2012.725488

Cooper, E.J. (2010). Gender Testing in Athletic Competitions-Human Rights Violations: Why Michael Phelps is Praised and Caster Semenya is Chastised. Journal of Gender, Race and Justice, 14, 233-264.

D'Angelo, C. y Tamburrini, C. (2013). Las atletas intersexuales y la equidad de género: ¿son sexistas las normas del COl?. Archivos de medicina del deporte: revista de la Federación Española de Medicina del Deporte y de la Confederación Iberoamericana de Medicina del Deporte, 154, 102-107.

Dimeo, P. (2007). A history of drug use in sport 1876-1976. Beyond good and evil. New York: Routledge. https://doi.org/10.4324/9780203003701

García Candau, J. (27 de septiembre, 2009). La historia de María (Jorge) Torremadé. Faro de Vigo, 2. Recuperado de: http://www.farodevigo.es/deportes/2009/09/28/historia-maria-jorgetorremade/372370.html

García Dauder, S. (2011). Las fronteras del sexo en el deporte: tecnologías, cuerpos sexuados y diferencia. Revista Internacional Interdisciplinar INTERthesis, 8(2), 1-19. https://doi.org/10.5007/1807-1384.2011v8n2p1 
Gleaves, J. (2015). Manufactured Dope: How the 1984 US Olympic Cycling Team Rewrote the Rules on Drugs in Sports. The International Journal of the History of Sport, 89-107. https://doi.org/10.1080/09523367.2014.958667

Hargreaves, J. (1994). Sporting Females: Critical Issues in the History and Sociology of women's Sport. London: Routledge.

Heggie, V. (2010). Testing sex and gender in sports; reinventing, reimagining and reconstructing histories. Endeavour, 34(4), 157-163. https://doi.org/10.1016/..endeavour.2010.09.005

Hercher, L. (2010). Gender Verification: A Term Whose Time has Come and Gone. Journal of Genetic Counselors, 19, 551-553. https://doi.org/10.1007/s10897-010-9323-z

Hernández, A. (7 de julio, 2014). Una mujer no puede competir con mujeres. Sinetiquetas.org. Recuperado de: https://sinetiquetas.org/2014/07/07/una-mujer-no-puede-competir-conmujeres/

Hoberman, J. (2005). Testosterone dreams. Rejuvenation, aphrodisiac, doping. Berkeley: University of California Press. https://doi.org/10.1525/9780520939783

IAAF (International Association of Athletics Federations). (2018) Eligibility regulations for the female classification (athletes with Differences of Sex Development). Recuperado de: https://www.documentcloud.org/documents/4449932-IAAF-EligibilityRegulations-for-the-Female.htm

Karkazis, K., Jordan-Young, R., Davis, G. \& Camporesi, S. (2012). Out of bounds? A Critique of the New Policies on Hyperandrogenism in Elite Female Athletes. The American Journal of Bioethics, 12(7), 3-16. https://doi.org/10.1080/15265161.2012.680533

La Vanguardia (3 de diciembre, 2008). Una transexual, entre los cerebros del ajedrez. La Vanguardia. Recuperado de: https://www.lavanguardia.com/vida/20081203/53592462132/unatransexual-entre-los-cerebros-del-ajedrez.html

Ladrón de Guevara, M. (6 de julio, 2015). Simplemente María o... Jordi. Transexuales en el deporte. Andaluciainformacion.es. Recuperado de: https://andaluciainformacion.es/andalucia/518027/simplemente-maria-ojordi-transexuales-en-el-deporte/

Leal, T. (18 de diciembre, 2014). Dora Ratjen. El atleta que el nazismo convirtió en mujer para ganar una medalla. Libertad Digital. Recuperado de: https://www.libertaddigital.com/deportes/mas-deporte/2014-12-18/doraratjen-el-atleta-al-que-el-nazismo-convirtio-en-mujer-para-ganar-unamedalla-1276536339/

Leruite, M.T., Martos, P. y Zabala, M. (2015). Análisis del deporte femenino español de competición desde la perspectiva de protagonistas clave. Retos: nuevas tendencias en educación física, deporte y recreación, 28, 3-8. https://doi.org/10.47197/retos.v0i28.34821

Ley $3 / 2007$, de 15 de marzo, reguladora de la rectificación registral de la mención relativa al sexo de las personas. «BOE» núm. 65, de 16 de marzo de 2007. Referencia: BOE-A-2007-5585 
Machado, C. y Fernández, J.M. (2015). La mujer en el olimpismo durante la época franquista. Materiales para la Historia del Deporte, 2, 207-219.

Manrique, J.C. (2014). Actividad física y juventud en el franquismo (1937-1961). Revista Internacional de Medicina y Ciencias de la Actividad Física y el Deporte, 14(55), 427-449.

Manrique, J.C. (2018). La actividad físico-deportiva franquista como intento socializador de la juventud y la mujer. Revista Internacional de Medicina y Ciencias de la Actividad Física y el Deporte, 18(70), 303-329. https://doi.org/10.15366/rimcafd2018.70.007

Meyerowitz, J. (1998). Sex change and the popular press. Historical notes on transsexuality in the United States, 1930-1955. Journal of Lesbian and Gay Studies, 4(2), 159-187. https://doi.org/10.1215/10642684-4-2-159

Monforte, J. y Úbeda-Colomer, J. (2019). 'Como una chica': un estudio provocativo sobre estereotipos de género en educación física. Retos: nuevas tendencias en educación física, deporte y recreación, 36, 74-79. https://doi.org/10.47197/retos.v36i36.68598

Parks, L. (2014). Sex Testing and the Maintenance of Western Femininity in International Sport. The International Journal of History of Sport, 31(13), 1557-1576. https://doi.org/10.1080/09523367.2014.927184

Reeser, J. (2005). Gender identity and sport: is the playing field level? British Journal of Sports Medicine, 39, 695-699. https://doi.org/10.1136/bjsm.2005.018119

Ritchie, I. (2003). Sex tested, gender verified: controlling female sexuality in the age of containment. Sport History Review, 34(1), 80-98. https://doi.org/10.1123/shr.34.1.80

Rivera, A. (21 de enero, 2019). Las personas transexuales podrán participar en competiciones ciclistas utilizando el nombre y género que sienten. Europapress. Recuperado de: https://www.europapress.es/sociedad/noticia-personas-transexualespodran-participar-competiciones-ciclistas-utilizando-nombre-generosienten-20190121135259.html

Ródenas, J.L. (2014). El atletismo español durante el gobierno de la UCD (19771982): radiografía de un deporte en evolución. En C. Navajas y D. Uturriaga (eds.), España en democracia: Actas del IV Congreso de Historia de nuestro tiempo (pp. 235-246) Logroño: Universidad de La Rioja.

Sullivan, C. (2011). Gender Verification and Gender Policies in Elite Sport: Eligibility and "Fair Play". Journal of Sport and Social Issues, 35(4), 400419. https://doi.org/10.1177/0193723511426293

Número de citas totales / Total references: 38 (100\%) Número de citas propias de la revista / Journal's own references: $2(5,2 \%)$

Rev.int.med.cienc.act.fís.deporte- vol. X - número X - ISSN: 1577-0354 